\title{
Metabolic changes, hypothalamo-pituitary-adrenal axis and oxidative stress after short-term starvation in healthy pregnant women
}

\author{
Sabrina Schraag1, Ursula von Mandach1, \\ Horst Schweer ${ }^{2}$ and Ernst Beinder ${ }^{1, *}$ \\ 1 Department of Obstetrics, Zurich University Hospital, \\ Zurich, Switzerland \\ 2 Department of Pediatrics, Philipps University Marburg, \\ Germany
}

\begin{abstract}
Aim: To compare metabolic effects and oxidative stress in pregnant and non-pregnant women after $12 \mathrm{~h}$ of fasting.

Methods: Twenty-six healthy women with uncomplicated singleton pregnancies between the $24^{\text {th }}$ and $28^{\text {th }}$ gestational week were recruited. After an overnight fast, venous blood samples and urine samples were tested for metabolic parameters characteristic for starvation, cortisol and oxidative stress products. Healthy non-pregnant women matched by age, body mass index and length of fasting comprised the control group.

Results: The metabolic parameters $\beta$-hydroxybutyrate and free fatty acids in blood and ketones in urine showed no differences in pregnant and non-pregnant women. However, the oxidative stress parameters, 8,12-iso- $\mathrm{PPF}_{2 \alpha}{ }^{-}$ $\mathrm{VI}$, isoprostanes and malondialdehyde were significantly higher in pregnant subjects, as was cortisol.

Conclusion: Healthy pregnant women are exposed to oxidative stress and activation of the hypothalamo-pituitary-adrenal axis, but not to metabolic changes resembling starvation during short fasting periods in comparison to non-pregnant healthy women.
\end{abstract}

Keywords: Fetal programming; isoprostanes; malondialdehyde; oxidative stress; pregnancy.

\section{Introduction}

Normal pregnancy is associated with a systemic inflammatory response. Acute phase reactants and inflammatory leukocytes are elevated, and both endothelial cells

\footnotetext{
${ }^{*}$ Corresponding author:

Dr. Ernst Beinder

Department of Obstetrics

Zurich University Hospital

Frauenklinikstr. 10

$\mathrm{CH}-8091$ Zurich

Switzerland

Tel.: +4144255 1111

Fax: +41442554430

E-mail: ernst.beinder@usz.ch
}

and thrombocytes are activated. Some of the metabolic changes during normal pregnancy have similarities with the metabolic syndrome which includes hyperlipidemia, insulin resistance and the activation of the clotting system $[12,17]$. Both systemic inflammation and the transient change into the metabolic syndrome in pregnancy were considered to be potential risk factors for both mother and fetus [19]. This view is supported by two important studies, the Framingham Heart Study and the National Health and Nutrition Examination Survey National Epidemiologic Follow-up Study (NHEFS) [9], where the rates of coronary heart disease were higher among multigravid women than among women who had never been pregnant.

Systemic inflammation is believed to be a physiological response towards "danger signals" within the organism. One possible explanation for inflammation in normal pregnancy is an increased level of oxidative stress. Oxidative stress is defined as an imbalance between free radical damage and antioxidant protection, caused by the presence of free radicals or radical-generating agents in concentrations that overwhelm natural radical-blocking or -scavenging mechanisms. Oxidative stress induces oxidative damage to biological substances such as lipids, DNA and proteins [3] and may be responsible for adverse changes in the unborn baby and thereby for effects of fetal programming and - in the long-term - for atherosclerosis and cardiovascular disease in the mother. Cardiovascular health can also be influenced by poor or unbalanced nutrition, increased urinary nitrogen excretion, increased plasma and urinary ketones and raised plasma levels of free fatty acids, the latter acting as a pro-oxidant substance. It is unclear whether the adverse effect of pregnancy (at least multiparity) on maternal cardiovascular health is the result of oxidative stress, activation of the HPA (hypothalamo-pituitary-adrenal)-axis, metabolic changes resembling starvation (catabolism) or a mixture thereof. We, therefore, examined whether pregnant women matched by age, body mass index (BMI) and duration of fasting are exposed to oxidative stress, activation of the HPA-axis and/or catabolic metabolism in comparison to non-pregnant women.

\section{Materials and methods}

A total of 27 healthy volunteers with uncomplicated singleton pregnancies between the $24^{\text {th }}$ and $28^{\text {th }}$ gestational week were recruited from the Department of Obstetrics at the University Hospital of Zurich in association with a routine fasting glucose 
control. The same number of healthy non-pregnant women matched by age group, BMl and length of fasting were recruited as a controls from the employees of the University Hospital. The non-pregnant controls were advised to fast for exactly the same time as their pregnant match. One pregnant woman was excluded from the study because gestational diabetes was diagnosed. Hence a total of 26 women were in each group, which allows a statistically significant interpretation of the results. Both the pregnant and the non-pregnant participants of the study were in good general health and had no disease or problems with their carbohydrate metabolism. Aside from standard prenatal vitamins or iron supplements they were not taking any medication. Each of the pregnant women gave birth to a healthy child. All volunteers gave written informed consent to participate in this study. The participation to this study was not financially rewarded.

To calculate the BMl of the pregnant women we used the pregravid weight. For randomization, we divided the women into four age- $(16-20,21-30,31-40$ and $41-45$ years) and BMI groups ( $<20$ underweight, 20-24.9 normal, 25-29.9 overweight, 30-39.9 obese). Age was calculated in completed years, whereas gestational age was measured accurate to a day. Gestational age was determined by the last menstrual period and corrected with early ultrasound, if necessary. Further details are given in Table 1.

Following an overnight fast, $25 \mathrm{~mL}$ of venous blood was taken and tested for free fatty acids (FFA), $\beta$-hydroxybutyrate, cortisol/ insulin-ratio and malondialdehyde. In addition, the subjects gave a morning urine sample which was used to measure ketones, creatinine, isoprostanes and 8,12-iso-iPF ${ }_{2 \alpha}-\mathrm{Vl}$. The same blood and urine samples were taken from non-pregnant controls at the same time in the morning after fasting overnight and skipping breakfast.

The study protocol was approved by the local Ethical Committee of the University of Zurich/Switzerland.

As oxidative stress markers we used malondialdehyde (MDA) in blood serum and 8,12-iso-iPF ${ }_{2 \alpha}-\mathrm{VI}$ and isoprostanes in urine corrected for creatinine. Markers for the metabolic status were cortisol/insulin ratio, serum-glucose, free fatty acids (FFA) and $\beta$-hydroxybutyrate. The blood samples were processed and frozen at $-20^{\circ} \mathrm{C}$ just after blood withdrawal and the urine samples just after collection, to avoid auto-oxidation or other processes that may have changed the concentration of the measured parameters.

All markers except MDA, isoprostanes and 8,12-iso-iPF ${ }_{2 \alpha}-\mathrm{VI}$ were measured at the Institute for Clinical Chemistry, University Hospital in Zurich.
Basal cortisol was determined in heparinized plasma by an electrochemiluminescence assay. $\beta$-hydroxybutyrate was measured by an enzymatic rate method with the use of $\beta$-hydroxybutyrate dehydrogenase. FFA in serum was determined by an enzymatic colorimetric-test and insulin with chemiluminescence assays. For the analysis of MDA, EDTA blood was separated by centrifugation at $4^{\circ} \mathrm{C}$ immediately after blood-withdrawal. The separated plasma samples were frozen and stored at $-20^{\circ} \mathrm{C}$ until analysis in the research laboratory of the Department of Obstetrics of the Zurich University Hospital using the Lipid Peroxidation Assay Kit from Calbiochem (Cat No 437634, Merck Biosciences, Darmstadt, Germany). The principle of this colorimetric assay is the reaction of a chromogenic reagent with MDA at $45^{\circ} \mathrm{C}$. One molecule of MDA condensates with two molecules of the reagent to yield a stable chromophore with a maximal absorbance at $586 \mathrm{~nm}$. Isoprostanes were analyzed in urine samples that were frozen at $-80^{\circ} \mathrm{C}$ in the Department of Pediatrics at PhilippsUniversity Marburg, Germany. The samples were processed as follows: Urine $(100 \mu \mathrm{L})$ was diluted with $200 \mu \mathrm{L}$ water and tetradeuterated $\left[{ }^{2} \mathrm{H}_{4}\right]-\mathrm{PGF}_{2 \alpha}(1 \mathrm{ng}$ in $10 \mu \mathrm{L}$ ethyl acetate) was added. After acidification with formic acid to $\mathrm{pH} 2.5$, the prostanoids were extracted twice with $1 \mathrm{~mL}$ ethyl acetate-hexane (70:30, $\mathrm{v} / \mathrm{v})$. After evaporation of the solvent, acetone $(80 \mu \mathrm{L}), N, N$-diisopropylethylamine $(7 \mu \mathrm{L})$ and pentafluorobenzyl bromide $(6 \mu \mathrm{L})$ were added. The mixture was allowed to react at $40^{\circ} \mathrm{C}$ for $25 \mathrm{~min}$. The dry sample was purified by thin layer chromatography (TLC, developing solvent: ethyl acetate-hexane 90:10, $\mathrm{v} / \mathrm{v})$. A broad zone $\left(R_{F} 0.03-0.39\right)$ was eluted with the TLC developing solvent $(1 \mathrm{~mL})$ and water $(50 \mu \mathrm{L})$ was added. After centrifugation, the ethyl acetate phase was withdrawn, the solvent was evaporated and the prostanoids derived with BSTFA: N,Obis(trimethylsilyl)trifluoro acetamide $\left(10 \mu \mathrm{L} ; 40^{\circ} \mathrm{C}, 1 \mathrm{~h}\right)$. A $1 \mu \mathrm{L}$ aliquot of this solution was injected.

For the analysis of 8,12 -iso-iPF $2 \alpha-\mathrm{VI}$ urine $(1 \mathrm{~mL})$ and $\left[{ }^{2} \mathrm{H}_{11}\right]-$ 8,12-iso-iPF ${ }_{2 \alpha}-\mathrm{VI}(1 \mathrm{ng})$ were acidified with formic acid to $\mathrm{pH} 2.5$ and the prostanoids were extracted twice with $3 \mathrm{~mL}$ of ethyl acetate-hexane $(70: 30, v / v)$. After evaporation of the solvent, acetonitrile $(80 \mu \mathrm{L})$, pentafluorobenzyl bromide $(7 \mu \mathrm{L})$ and $N, N$ diisopropylethylamine $(25 \mu \mathrm{L})$ were added. The mixture was allowed to react at $40^{\circ} \mathrm{C}$ for $25 \mathrm{~min}$. The dry sample was purified by TLC (developing solvent: ethyl acetate-methanol 100:3, v/v). The zone with an $R_{F}$ 0.2-0.33 was eluted with TLC ethylacetate $(800 \mu \mathrm{L})$ and water $(50 \mu \mathrm{L})$ was added. Further sample preparation was as described for the isoprostanes.

The concentration of each parameter in the prepared urine samples was determined using gas chromatography/tandem mass spectrometry (GC/MS/MS). A Finnigan MAT TSQ700 tan-

Table 1 Fasting duration, age, BMI, gestational and ethnical characteristics of the tested women.

\begin{tabular}{llll}
\hline & Pregnant $(\mathrm{n}=26)$ & Non-pregnant $(\mathrm{n}=26)$ & $\mathrm{P}$ \\
\hline Fasting time $(\mathrm{h})$ & $11.76 \pm 3.07$ & $11.84 \pm 2.96$ & $\mathrm{NS}(0.80)$ \\
Age (years) & $28.35 \pm 4.73$ & $27.58 \pm 4.98$ & $\mathrm{NS}(0.67)$ \\
BMI & $22.57 \pm 3.43$ & $22.13 \pm 3.34$ & $\mathrm{NS}(0.58)$ \\
Gestational week & $26.19 \pm 0.97$ & - & - \\
Parity & $1.73 \pm 0.78$ & $0.19 \pm 0.69$ & $\mathrm{~S}(<0.0001)$ \\
Caucasian & 19 & 26 & $\mathrm{NS}$ \\
Asian & 4 & - & \\
Afro-Caribbean & 1 & - & \\
Oriental & 2 & - & \\
\hline
\end{tabular}

Mean $\pm \mathrm{SD}$; $\mathrm{P}$, denotes significance $(<0.05)$ between pregnant and non-pregnant. 
dem mass spectrometer equipped with a Varian 3400 gas chromatograph and a CTC A200S autosampler (Finnigan MAT, Bremen, Germany) was employed. Gas chromatography of prostanoid derivatives was carried out on J\&W DB-1 (15 $\mathrm{m} \times 0.25 \mathrm{~mm}$ I.D., $0.25 \mu \mathrm{m}$ film thickness) capillary column (MasCom, Bremen, Germany) in the splitless mode at an inlet pressure of $100 \mathrm{kPa}$. The oven temperature program for all prostanoids analyzed was as follows: an initial temperature of $100^{\circ} \mathrm{C}$ was held for $2 \mathrm{~min}$, and then increased at $30^{\circ} / \mathrm{min}$ to $280^{\circ} \mathrm{C}$ and at $5 \% \mathrm{~min}$ to $310^{\circ} \mathrm{C}$. This temperature was held for $2 \mathrm{~min}$. The mass spectrometer conditions were: interface temperature $300^{\circ} \mathrm{C}$, source temperature $150^{\circ} \mathrm{C}$, methane $\mathrm{Cl}$ gas pressure $50 \mathrm{~Pa}$, electron energy $70 \mathrm{eV}$, emission current $0.4 \mathrm{~mA}$, conversion dynode $15 \mathrm{kV}$, electron multiplier $2000 \mathrm{~V}$ and the collision cell pressure was $0.2 \mathrm{~Pa}$. For the isoprostanes the collision energy was $12 \mathrm{eV}$ and the daughter ions used for quantification were $\left[\mathrm{P}-3\left(\mathrm{CH}_{3}\right)_{3} \mathrm{SiOH}\right]^{-}(\mathrm{m} / \mathrm{z} 569.3 \rightarrow \mathrm{m} / \mathrm{z} 299.3$ for the endogenous isoprostanes, $\mathrm{m} / \mathrm{z} 573.3 \rightarrow \mathrm{m} / \mathrm{z} 303.2$ for the tetradeuterated internal standard). For $8,12-i$ so- $^{-} \mathrm{PFF}_{2 \alpha}-\mathrm{VI}$ the parent ions of the characteristic fragment $\mathrm{C}_{6} \mathrm{H}_{7} \mathrm{O}_{2}^{-}(\mathrm{m} / \mathrm{z} 111.0)$ were used: $\mathrm{m} / \mathrm{z}$ 569.3 for the endogenous 8,12-iso-iPF ${ }_{2 \alpha}-\mathrm{Vl}$ and $\mathrm{m} / \mathrm{z} 580.4$ for the deuterated internal standard. Collision energy was $28 \mathrm{eV}$. The urinary 8,12-iso-iPF $\mathrm{PF}_{2 \alpha} \mathrm{VI}$ and isoprostane levels were corrected for creatinine values to eliminate influences of renal function on the result.

Statistical difference between the two groups was determined by Mann-Whitney U-Test. We used StatView for Windows, version 5.0.1 (SAS Institute Inc. Copyright, (C) 1992-1998, www.statview.com). A P-value $<0.05$ was considered statistically significant. The graphical data are presented with scatterplots and median values.

\section{Results}

The groups were similar regarding fasting time, BMI, and age (Table 1). The concentrations of circulating oxidative and metabolic parameters are summarized in Table 2. The women's individual BMI did not correlate with the measured concentration of oxidative stress products. Regarding cortisol, we found a slight, but not a significant increase with greater BMI in pregnant women. No significant correlation was observed for non-pregnant subjects.

Metabolic parameters: Serum-insulin tended to be higher in pregnant women, but the difference did not

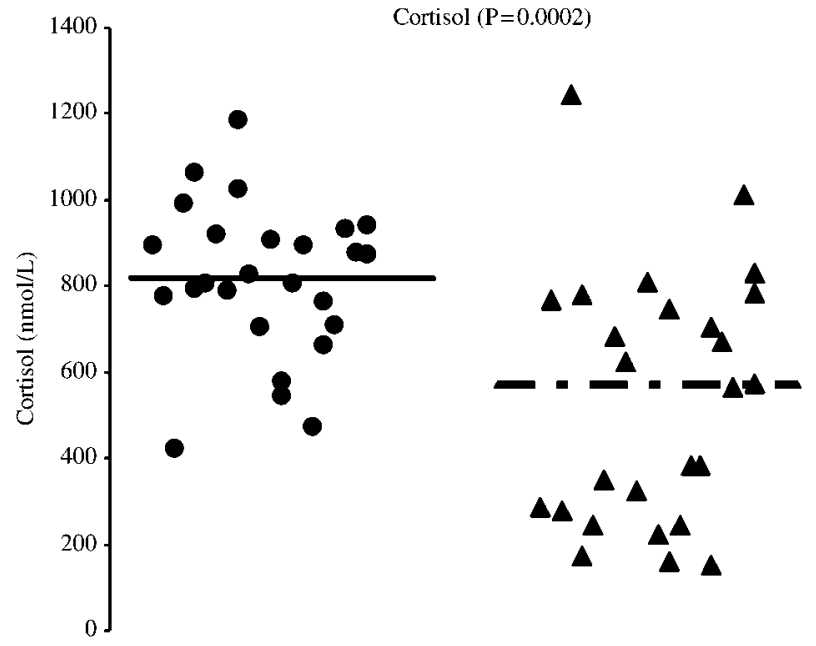

Figure 1 Cortisol serum levels after having skipped breakfast. Pregnant women during their second trimester have significantly elevated cortisol levels compared to non-pregnant women. Circles $=$ pregnant, pyramids $=$ controls, continuous line $=$ median of pregnant, dashed line $=$ median of controls.

reach significance (Table 2). All of the metabolic parameters (serum-glucose, free fatty acids (FFA), $\beta$-hydroxybutyrate and the cortisol/insulin-ratio) were not significant (Table 2).

HPA-axis: We observed a significant elevation of cortisol in pregnant subjects $(\mathrm{P}=0.0002)$ compared to nonpregnant subjects (Figure 1).

Oxidative stress parameters: The tested oxidative stress parameters, namely isoprostanes $(P<0.0001)$, 8,12-iso-iPF ${ }_{2 \alpha}-\mathrm{VI}(\mathrm{P}=0.0008)$ (Figure 2), and malondialdehyde $(P<0.0001)$ (Figure 3$)$, were significantly elevated in pregnant women.

\section{Comment}

We could show that after a short starvation, all of our tested parameters of oxidative stress as well as serum cortisol levels were significantly elevated in pregnant women in the late second trimester in comparison to

Table 2 Duration of fasting and concentration of parameters after "skipped breakfast".

\begin{tabular}{|c|c|c|c|}
\hline & Pregnant $(n=26)$ & Non-pregnant $(n=26)$ & $\mathrm{P}$ \\
\hline Glucose ( $\mu \mathrm{mol} / \mathrm{L})$ & $4.32 \pm 0.31$ & $4.50 \pm 0.33$ & NS (0.07) \\
\hline$\beta$-hydroxybutyrate $(\mu \mathrm{mol} / \mathrm{L})$ & $171.81 \pm 86.35$ & $189.81 \pm 73.72$ & NS (0.13) \\
\hline $\mathrm{FFA}(\mu \mathrm{mol} / \mathrm{L})$ & $391.42 \pm 153.21$ & $465.89 \pm 254.81$ & NS (0.55) \\
\hline Cortisol (nmol/L) & $813 \pm 178.28$ & $538.69 \pm 291.61$ & $S(0.0002)$ \\
\hline Insulin (mIU/L) & $6.92 \pm 5.05$ & $4.81 \pm 4.22$ & NS (0.062) \\
\hline C/I-ratio $\left(10^{3}\right)$ & $28.13 \pm 22.77$ & $24.51 \pm 20.93$ & NS (0.37) \\
\hline Malondialdehyde ( $\mu \mathrm{mol} / \mathrm{L})$ & $4.52 \pm 0.77$ & $2.88 \pm 0.52$ & $S(<0.0001)$ \\
\hline $8,12-i s o-\mathrm{iPF}_{2 \alpha}-\mathrm{VI}(\mathrm{ng} / \mathrm{mg})^{*}$ & $13.69 \pm 6.46$ & $8.31 \pm 5.05$ & $S(0.0008)$ \\
\hline Isoprostanes (ng/mg) & $78.15 \pm 23.72$ & $59.92 \pm 40.77$ & $S(<0.0001)$ \\
\hline
\end{tabular}

Mean \pm SD; $P$, denotes significance $(<0.05)$ between pregnant and non-pregnant. ${ }^{*}$ Corrected for creatinine. 


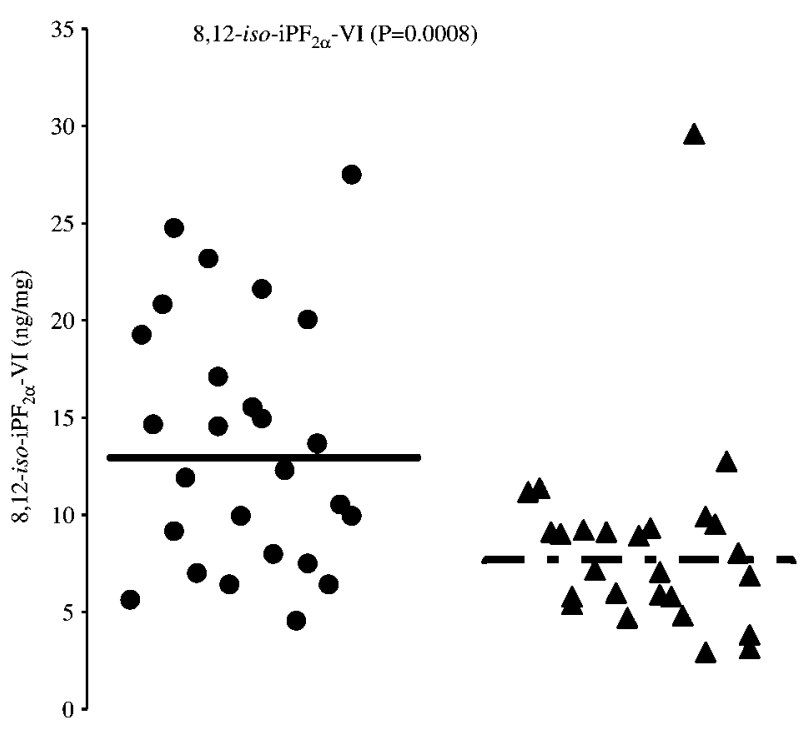

Figure 2 8,12-iso-iPF ${ }_{2 \alpha}-\mathrm{VI}$ levels in urine after fasting. Pregnant women have significantly elevated levels, which is due to the greater oxidative stress.

Circles $=$ pregnant, pyramids $=$ controls, continuous line $=$ median of pregnant, dashed line $=$ median of controls.

healthy non-pregnant controls. Metabolic parameters indicative of catabolism, however, were not altered.

The level of oxidative stress may vary considerably throughout day and night and depends on the interval since the last food intake. This might be the reason for the conflicting results in the literature regarding the occurrence of oxidative stress in normal pregnancy and in preeclampsia $[4,7,15]$. Consequently, the metabolic parameters of catabolism and plasma levels of cortisol vary significantly if they are not measured under standard conditions. The measurements in our study were conducted in a healthy pregnant population matched with the non-pregnant controls for BMI, age and period of fasting. Moreover, serum malondialdehyde and 8,12-iso$\mathrm{iPF}_{2 \alpha}-\mathrm{VI}$ and isoprostanes in urine corrected for creatinine are the most reliable markers for oxidative stress $[2,15$, 16]. We measured oxidative stress, cortisol and the metabolic parameters the morning after an overnight fast, because this interval allows for standardized measurements and represents a common metabolic situation occurring in many pregnant women who decide to skip breakfast due to personal preference, work overload, or for laboratory testing. Therefore, we believe that our results truly reflect increased oxidative stress and an activation of the HPA-axis in pregnant women in comparison to non-pregnant women under physiological conditions.

It is well known that both oxidative stress and increased plasma cortisol levels are risk factors for atherosclerosis and cardiovascular disease [22]. Interestingly, women having six or more pregnancies show a consistently increased risk of coronary heart and cardiovascular disease [9]. However, the same tendency was observed with a lower number of pregnancies [9]. Oxidative stress in pregnancy, in combination with an activation of the HPA-axis, may induce micro-damage to maternal arteries and, therefore, might serve as a possible link between multigravidity and adverse maternal long term outcome. However, the mother may not be the only one affected

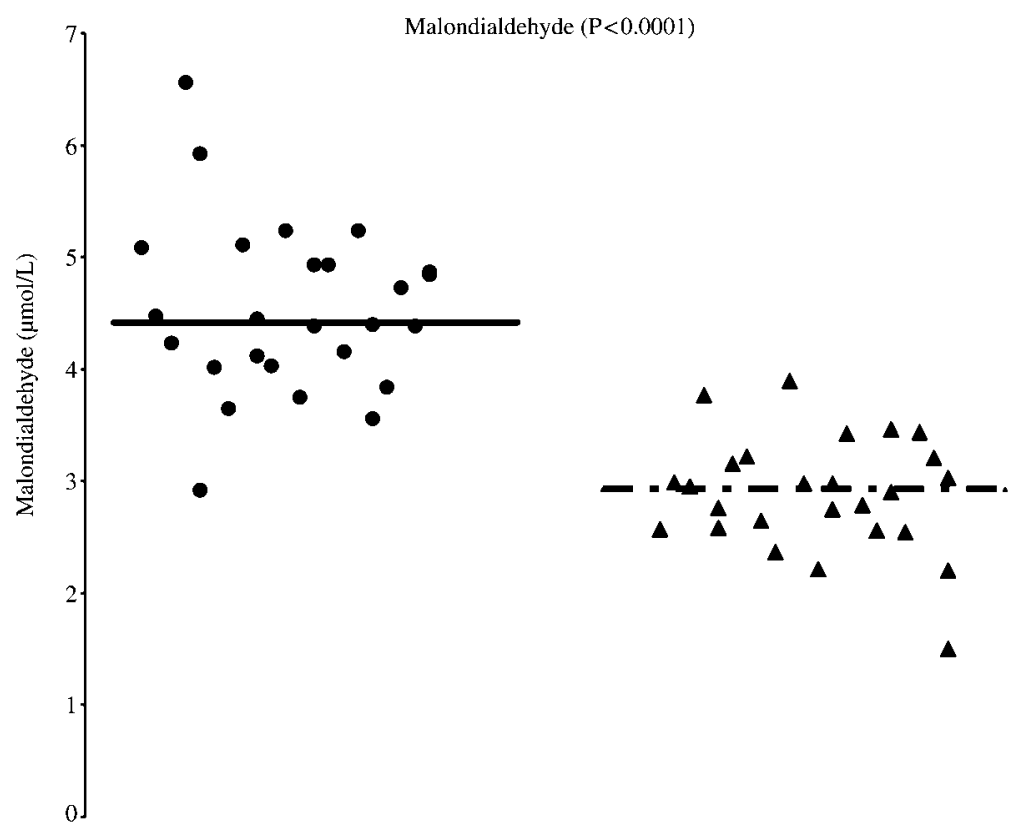

Figure 3 Malondialdehyde concentration in serum after having skipped breakfast. Pregnant women having significantly elevated malondialdehyde levels compared to non-pregnant women.

Circles $=$ pregnant, pyramids $=$ controls, continuous line $=$ median of pregnant, dashed line $=$ median of controls. 
by oxidative stress and an activated HPA axis in pregnancy, because it was hypothesized that unfavorable influences during pregnancy can have a long lasting impact on the unborn baby (Barker's hypothesis of "Fetal Programming" [1, 10]). The exact mechanisms responsible for fetal programming are unknown. It has been proven that women with poor nutrition and a stressful pregnancy have a greater risk of bearing an underweight baby that could be affected by the aforementioned diseases [5, 13]. Epidemiological studies on the effect of severe maternal long term starvation during the Dutch famine in World War II showed that this is associated with decreased glucose tolerance and altered endothelial function in the offspring, especially when they became obese [13]. However, this association could only be shown in the Dutch famine study, but not in the Leningrad siege study [21].

There is overwhelming evidence that suboptimal conditions experienced during intrauterine life could affect critical fetal systems. Elevated cortisol levels and oxidative stress may be the agents of intrauterine mal-programming of endocrine and circulatory regulatory systems with the consequence of an increased susceptibility for diseases in later life [8, 20]. Oxidative stress in pregnancy may, therefore, affect the health of mother and fetus alike.

Important substances for the diagnosis of oxidative stress are the isoprostanes. They are prostaglandin $\mathrm{F}_{2}-$ like compounds that are generated in vivo in humans by non-enzymatic free radical-induced peroxidation of arachidonic acid and show high stability [22]. Isoprostanes have been shown to be elevated in a number of disorders that are possibly associated with oxidative stress [16].

One of the major compounds of this group, 8,12-iso$\mathrm{iPF}_{2 \alpha}-\mathrm{VI}$, is a chemically stable, free-radical catalyzed isoprostane that reflects lipid peroxidation. After free-radical attack, it is cleaved from the plasma membrane into the blood and excreted in the urine. This substance has particular importance as an indicator of oxidative stress in vivo.

Interestingly, in our study, metabolic parameters indicative of catabolism were not elevated in the late second trimester in comparison to non-pregnant controls after a mean fasting time of $12 \mathrm{~h}$. Free fatty acids induce oxidative stress and, therefore, catabolism is thought to be a cardiovascular risk factor. Catabolism in our study is unlikely to be the reason for the elevated oxidative stress parameters. Metzger et al. [6] described the socalled "accelerated starvation" after a fasting period of $18 \mathrm{~h}$ in the third trimester of pregnancy. After $12 \mathrm{~h}$ of fasting, the results resemble ours in so far that they could not show catabolic changes in pregnant women. As far as carbohydrate metabolism is concerned, other studies found similar results to those found by Metzger et al. with catabolism and fat mobilization after the first $12 \mathrm{~h}$ of starvation $[11,14,18]$. Because of ethical reasons, prolon- gation of the fasting time was not contrivable in our study.

To the best of our knowledge, this is the first study to simultaneously examine metabolic parameters characteristic for catabolism, plasma basal cortisol and oxidative stress under standardized conditions in pregnancy. Each of the three parameters serves as a risk factor for cardiovascular disease. We were able to show that oxidative stress parameters and cortisol levels are elevated during normal pregnancy after "skipping breakfast" in comparison to non-pregnant controls. This elevation is not accompanied by metabolic signs of starvation. We hypothesize that the combination of oxidative stress and an activation of the HPA-axis is the link between the long term adverse effects of pregnancy (at least in multiparity) for cardiovascular diseases and (if further increased) might induce an adverse intrauterine environment that can have unfavorable and long-lasting effects on the fetus. Our study, therefore, serves as a theoretical basis that antioxidants might be useful to prevent long term adverse effects of multiparity on maternal health.

\section{Acknowledgements}

Alexandra Dolder is acknowledged for her excellent laboratory work.

\section{References}

[1] Barker DJ. Fetal origins of coronary heart disease. Br Med J. 1995;311:171-4.

[2] Basu S. Isoprostanes: novel bioactive products of lipid peroxidation. Free Radic Res. 2004;38:105-22.

[3] Droge W. Free radicals in the physiological control of cell function. Physiol Rev. 2002;82:47-95.

[4] Ishihara O, Hayashi M, Osawa H, Kobayashi K, Satoru T, Vessby B, et al. Isoprostanes, prostaglandins and tocopherols in preeclampsia, normal pregnancy and non-pregnancy. Free Radic Biol Med. 2004;38:913-8.

[5] Louey S, Thornburg KL. The prenatal environment and later cardiovascular disease. Early Hum Dev. 2005;81:74551.

[6] Metzger BE, Ravnikar V, Vileisis RA, Freinkel N. "Accelerated starvation" and the skipped breakfast in late normal pregnancy. Lancet. 1982;8272:588-92.

[7] Morris JM, Gopaul NK, Endresen MJ, Knight M, Linton EA, Dhir S, et al. Circulating markers of oxidative stress are raised in normal pregnancy and preeclampsia. Br J Obstet Gynaecol. 1998;105:1195-9.

[8] Nathanielsz PW, Berghorn KA, Derks JB, Giussani DA, Docherty C, Unno N, et al. Life before birth: effects of cortisol on future cardiovascular and metabolic function. Acta Paediatr. 2003;92:766-72.

[9] Ness RB, Harris T, Cobb J, Flegal KM, Kelsey JL, Balanger $A$, et al. Number of pregnancies and the subsequent risk of cardiovascular disease. N Engl J Med. 1993;328:152833. 
[10] Paneth N, Susser M. Early origin of coronary heart disease (the "Barker hypothesis") (editorial). Br Med J. 1995;310: 411-2.

[11] Phelps RL, Metzger BE, Freinkel N. Carbohydrate metabolism in pregnancy XVII. Diurnal profiles of plasma glucose, insulin, free fatty acids, trigycerides, cholesterol, and individual amino acids in late normal pregnancy. Am J Obstet Gynecol. 1981;140:730-6.

[12] Potter JM, Nestel PJ. The hyperlipidemia of pregnancy in normal and complicated pregnancies. Am J Obstet Gynecol. 1979;133:165-70.

[13] Ravelli AC, van der Meulen JH, Michels RP, Osmond C, Barker DJ, Hales CN, et al. Glucose tolerance in adults after prenatal exposure to famine. Lancet. 1998;351:1737.

[14] Ravnikar V. Is there a risk of "accelerated starvation" in normal pregnancy? (Abstract) Diabetes. 1978;27:463.

[15] Regan CL, Levine RJ, Baird DD, Ewell MG, Martz KL, Sibai $\mathrm{BM}$, et al. No evidence for lipid peroxidation in severe preeclampsia. Am J Obstet Gynecol. 2001;185:572-8.

[16] Roberts LJ, Morrow JD. Measurement of $F_{2}$-Isoprostanes as an index of oxidative stress in vivo. Free Radic Biol Med. 2000;28:505-13.

[17] Sacks GP, Studena K, Sargent IL, Redman CW. Normal pregnancy and preeclampsia both produce inflammatory changes in peripheral blood leukocytes akin to those of sepsis. Am J Obstet Gynecol. 1998;179:80-6.

[18] Saleh AK, Al-Muhtaseb N, Gumaa KA, Mubarak A, Shaker MS. Maternal, amniotic fluid and cord blood metabolic profile in normal pregnant and gestational diabetics during recurrent withholding of food. Horm Metab Res. 1989;21: 507-13.

[19] Sattar N, Greer IA. Pregnancy complications and maternal cardiovascular risk: opportunities for intervention and screening? Br Med J. 2002;325:157-60.

[20] Seckl JR, Cleasby M, Nyirenda MJ. Glucocorticoids, 11ßhydroxysteroid dehydrogenase, and fetal programming. Kidney International. 2000;57:1412-7.

[21] Stanner SA, Bulmer K, Andrès C, Lantseva OE, Borodina $\mathrm{V}$, Poteen VV, et al. Does malnutrition in utero determine diabetes and coronary heart disease in adulthood? Results from the Leningrad siege study, a cross sectional study. Br Med J. 1997;315:1342-8.

[22] Steinberg D, Parthasarathy S, Carew T, Khoo JC, Witztum JL. Beyond cholesterol. Modifications of low-density lipoprotein that increase its atherogenicity. $\mathrm{N}$ Engl J Med. 1989;320:915-24.

Received January 28, 2007. Revised March 25, 2007. Accepted March 28, 2007. Published online on June 1, 2007. 